\title{
Dravet Syndrome Patient-Derived Neurons Suggest a Novel Epilepsy Mechanism
}

\author{
Yu Liu, MD, PhD, ${ }^{1}$ Luis F. Lopez-Santiago, $\mathrm{PhD}^{2}{ }^{2}$ Yukun Yuan, $\mathrm{PhD},{ }^{2}$ \\ Julie M. Jones, MS, ${ }^{3}$ Helen Zhang, MS, ${ }^{1}$ Heather A. O'Malley, PhD, ${ }^{2}$ \\ Gustavo A. Patino, PhD, 1,2,4 Janelle E. O’Brien, PhD, ${ }^{3}$ Raffaella Rusconi, PhD, ${ }^{2}$ \\ Ajay Gupta, MD, ${ }^{5}$ Robert C. Thompson, $\mathrm{PhD}^{4,6}$ \\ Marvin R. Natowicz, MD, PhD, 5,7,8,9,10 Miriam H. Meisler, PhD, 3,4 \\ Lori L. Isom, PhD, ${ }^{2,4}$ and Jack M. Parent, MD ${ }^{1,4,11}$
}

Objective: Neuronal channelopathies cause brain disorders, including epilepsy, migraine, and ataxia. Despite the development of mouse models, pathophysiological mechanisms for these disorders remain uncertain. One particularly devastating channelopathy is Dravet syndrome (DS), a severe childhood epilepsy typically caused by de novo dominant mutations in the SCN1A gene encoding the voltage-gated sodium channel $\mathrm{Na}_{v} 1.1$. Heterologous expression of mutant channels suggests loss of function, raising the quandary of how loss of sodium channels underlying action potentials produces hyperexcitability. Mouse model studies suggest that decreased $\mathrm{Na}_{v} 1.1$ function in interneurons causes disinhibition. We aim to determine how mutant SCN1A affects human neurons using the induced pluripotent stem cell (iPSC) method to generate patient-specific neurons.

Methods: Here we derive forebrain-like pyramidal- and bipolar-shaped neurons from 2 DS subjects and 3 human controls by iPSC reprogramming of fibroblasts. DS and control iPSC-derived neurons are compared using whole-cell patch clamp recordings. Sodium current density and intrinsic neuronal excitability are examined.

Results: Neural progenitors from DS and human control iPSCs display a forebrain identity and differentiate into bipolar- and pyramidal-shaped neurons. DS patient-derived neurons show increased sodium currents in both bipolar- and pyramidal-shaped neurons. Consistent with increased sodium currents, both types of patient-derived neurons show spontaneous bursting and other evidence of hyperexcitability. Sodium channel transcripts are not elevated, consistent with a post-translational mechanism.

Interpretation: These data demonstrate that epilepsy patient-specific iPSC-derived neurons are useful for modeling epileptic-like hyperactivity. Our findings reveal a previously unrecognized cell-autonomous epilepsy mechanism potentially underlying DS, and offer a platform for screening new antiepileptic therapies.

ANN NEUROL 2013;74:128-139

$\mathrm{N}$ euronal ion channelopathies due to genetic mutations comprise a constellation of central and peripheral nervous system disorders that manifest as epilepsy, migraine, ataxia, abnormal pain sensation, or anosmia (reviewed in
Catterall et $\mathrm{al}^{1}$ and Kullmann and Waxman ${ }^{2}$ ). Although the affected genes and involved neuronal subtypes are often known, the pathophysiological mechanisms underlying most of these disorders remain poorly understood. One

View this article online at wileyonlinelibrary.com. DOI: 10.1002/ana.23897

Received Oct 16, 2012, and in revised form Feb 25, 2013. Accepted for publication Mar 1, 2013.

Address correspondence to Dr Parent, 5021 BSRB, 109 Zina Pitcher Place, Ann Arbor, Ml 48109-2200. E-mail: parent@umich.edu

Current address for Dr Rusconi: German Center for Neurodegenerative Disease, Bonn, Germany

From the ${ }^{1}$ Departments of Neurology, ${ }^{2}$ Pharmacology, and ${ }^{3}$ Human Genetics, and ${ }^{4}$ Neuroscience Graduate Program, University of Michigan Medical Center, Ann Arbor, Ml; ${ }^{5}$ Neurological Institute, Cleveland Clinic Lerner College of Medicine, Cleveland, OH; ${ }^{6}$ Department of Psychiatry, University of Michigan Medical Center, Ann Arbor, Ml; ${ }^{7}$ Genomic Medicine, ${ }^{8}$ Pediatric, and ${ }^{9}$ Pathology and Laboratory Medicine Institutes, Cleveland Clinic, Cleveland, $\mathrm{OH}$; and ${ }^{10}$ Lerner College of Medicine, Cleveland Clinic, Cleveland, $\mathrm{OH}_{;}{ }^{11}$ VA Ann Arbor Healthcare System, Ann Arbor, MI 
such disorder is Dravet syndrome (DS), a devastating childhood-onset epilepsy typically characterized by multiple pharmacoresistant seizure types, cognitive regression, ataxia, and increased mortality. ${ }^{3,4}$ In $>70 \%$ of cases, DS is caused by de novo mutations in the SCN1A gene which encodes the $\alpha$ subunit of the neuronal voltage-gated sodium channel $\mathrm{Na}_{\mathrm{v}} 1.1 .{ }^{5,6} \quad$ Voltage-gated sodium channels underlie action potentials in excitable cells such as neurons and myocytes. DS is thought to be caused by SCN1A haploinsufficiency, ${ }^{7-9}$ raising the question of how a partial loss of sodium channel expression leads to neuronal hyperexcitability.

The effects of human DS SCN1A mutations or deletions of mouse Scn 1a have been studied in heterologous expression systems and transgenic mouse models, ${ }^{10-13}$ but not in human neurons. The opportunity to study the effects of neuronal ion channel gene mutations in patient-specific neurons is now available using the induced pluripotent stem cell (iPSC) technique. This method involves reprogramming somatic cells, such as fibroblasts, using defined genetic factors to generate PSCs, and it has been accomplished in mice, humans, and other species. ${ }^{14-17}$ Recently, the effects of ion channel gene mutations have been studied in patientspecific cardiac myocytes generated from iPSCs, and these reports suggest that the patient-specific cells effectively model aspects of genetic cardiac arrhythmias. ${ }^{18-20}$ However, this approach has not yet been applied to the study of human neuronal ion channelopathies.

Here we describe a splice donor site mutation ${ }^{21}$ in the SCN1A gene of a 7-year-old DS patient. This mutation results in an abnormal transcript that skips exon 14 . Expression of the mutant SCN1A cDNA in heterologous systems resulted in little to no $\mathrm{Na}_{\mathrm{v}} 1.1$ protein and no detectable sodium current. We established human iPSC lines from the fibroblasts of this DS patient, 1 additional DS patient with a nonsense mutation, and 3 human controls. We generated forebrain-like neurons from DS and control iPSCs and found increased sodium current density in pyramidal- and bipolar-shaped neurons from both DS patients. This increased sodium current density was associated with spontaneous bursting and other evidence of epileptic-like hyperexcitability in DS patient neurons. Our results using patient-derived neurons suggest an unexpected pathophysiological mechanism underlying epilepsy in DS, and demonstrate that iPSC models of DS and other neuronal ion channelopathies will be useful for exploring disease pathogenesis and identifying novel therapies.

\section{Subjects and Methods}

\section{Expression Vectors}

Human $\mathrm{Na}_{\mathrm{v}} 1.1$ cDNA in pCDM8 was described previously. ${ }^{22}$ The IVS14+3A $>$ T mutation was introduced into this vector using the Quick Change XL Kit (Stratagene, La Jolla, CA). Bacteria-containing plasmids were grown at low temperature to reduce rearrangements as described. ${ }^{22}$ Wild-type and mutant cDNAs were sequenced completely before use.

\section{Western Blot Analysis}

tsa-201 cells (maintained at $37^{\circ} \mathrm{C}$ with $5 \% \mathrm{CO}_{2}$ ) were cultured with Dulbecco modified Eagle medium (DMEM)/F12 medium supplemented with $10 \%$ heat-inactivated fetal bovine serum and $100 \mathrm{U}+100 \mu \mathrm{g} / \mathrm{ml}$ penicillin/streptomycin (all from Invitrogen, Carlsbad, CA) in poly-D-Lysine-pretreated $10 \mathrm{~cm}$ dishes. Cells were mock-transfected or transfected at 60 to $70 \%$ confluency with pCDM8 vectors encoding wild-type or mutant human $\mathrm{Na}_{\mathrm{v}} 1.1$ cDNA using the $\mathrm{CaPO}_{4}$ method as described. ${ }^{22}$ Transfected cells at $100 \%$ confluence were prepared for Western blot as described. ${ }^{23}$ Proteins were separated by sodium dodecyl sulfate polyacrylamide gel electrophoresis on 5\% polyacrylamide gels, transferred to nitrocellulose, and analyzed using the SNAP i.d. apparatus (Millipore, Billerica, MA) per the manufacturer's instructions. Primary antibodies were rabbit anti-pan sodium channel (1:1,000; Sigma, St Louis, MO) and mouse anti- $\alpha$ tubulin monoclonal (1:5,000; Cedarlane Laboratories, Burlington, NC). Secondary antibodies were horseradish peroxidaseconjugated goat antirabbit or antimouse (1:2,000; Pierce, Rockford, IL). Immunoreactive signals were quantified using ImageJ (National Institutes of Health) and normalized to $\alpha$-tubulin loading control.

\section{Electrophysiological Recordings}

For recordings of cultured oocytes, human $\mathrm{Na}_{\mathrm{v}} 1.1$ wild-type or IVS14+3A $>$ T mutant cDNAs in pCDM8 were linearized with ScaI and mRNA synthesized using the T7 mMessage mMachine RNA Synthesis Kit (Ambion, Austin, TX). Transcripts were injected into Xenopus oocytes, and expressed sodium currents were analyzed by 2 -electrode voltage clamp as described. ${ }^{24}$ Published methods for mRNA analysis, frog maintenance, and oocyte retrieval were used. ${ }^{24}$ iPSC-derived neurons were analyzed by whole-cell patch clamp recordings. Voltage clamp recordings were performed in the standard whole-cell configuration, using previously described conditions. ${ }^{25}$ Isolated sodium currents were recorded from single neurons (bipolar or pyramidal) at room temperature $\left(21-22^{\circ} \mathrm{C}\right)$ in the presence of a bath solution containing (in millimolar): $120 \mathrm{NaCl}, 1 \mathrm{BaCl}_{2}, 2$ $\mathrm{MgCl}_{2}, 0.2 \mathrm{CdCl}_{2}, 1 \mathrm{CaCl}_{2}, 10$ HEPES, $20 \mathrm{TEA}-\mathrm{Cl}$, and 10 glucose $(\mathrm{pH}=7.35$ with $\mathrm{CsOH}$; osmolarity $=300-305$ mosm $)$. Fire-polished patch pipettes were filled with an internal solution containing (in millimolar): $1 \mathrm{NaCl}, 150 \mathrm{~N}$-methyl-D-glucamine, 10 ethyleneglycoltetraacetic acid (EGTA), $2 \mathrm{MgCl}_{2}, 40$ HEPES, and 25 phosphocreatine-tris, $2 \mathrm{MgATP}, 0.02 \mathrm{Na}_{2} \mathrm{GTP}$, 0.1 leupeptin $\left(\mathrm{pH}=7.2\right.$ with $\left.\mathrm{H}_{2} \mathrm{SO}_{4}\right)$. All recordings were performed within 10 to 120 minutes after the culture medium was replaced by bath recording solution and the dish with cells was placed on the recording setup. For current-clamp recordings of action potentials in iPSC-derived neurons, patch pipettes were filled with internal solution consisting of (in millimolar): 135 K-gluconate, $4 \mathrm{NaCl}, 0.5 \mathrm{CaCl}_{2}, 10 \mathrm{HEPES}, 5 \mathrm{EGTA}, 2 \mathrm{Mg}$ - 
ATP, and 0.4 GTP ( $\mathrm{pH}=7.3$, adjusted with $\mathrm{KOH}$ ). iPSCs were bathed in a solution consisting of (in millimolar): 115 $\mathrm{NaCl}, 2.5 \mathrm{KCl}, 1 \mathrm{MgCl}_{2}, 1.25 \mathrm{KH}_{2} \mathrm{PO}_{4}, 26 \mathrm{NaHCO}_{3}, 2$ $\mathrm{CaCl}_{2}, 10 \mathrm{HEPES}$, and $10 \mathrm{D}$-glucose $(\mathrm{pH}=7.4$, adjusted with $\mathrm{NaOH}$ ). Action potentials were evoked from resting membrane potential by injection of a series of increasing 1-millisecond depolarizing currents $(0.02 \mathrm{nA}$ increments $)$ beginning at subthreshold level and rising until action potentials were consistently generated. The threshold potential was defined as the membrane potential at which these ascending current steps first initiated an action potential, and was measured at the end of the depolarizing pulse and the initiation point of the action potential. Repetitive spiking was evoked by injection of a 1,500-millisecond depolarizing current $(0.02 \mathrm{nA})$ from a holding potential at resting levels.

\section{Generation of iPSCs and Patient-Specific Neurons}

DS patient and control skin biopsy-derived fibroblast cultures were obtained from the Cleveland Clinic under a protocol approved by the institutional review board. Retroviral vectors (pMXs-Oct3/4, pMXs-Sox2, pMXs-Klf4, pMXs-c-Myc) were obtained from Addgene (Cambridge, MA), and retroviral stocks were generated using GP2-293 packaging cells (Clontech, Mountain View, CA). To generate iPSCs from human fibroblasts (cultured in DMEM plus 10\% fetal calf serum), 2 rounds of viral transduction were performed on 30,000 fibroblasts, and cells were incubated with virus for another 48 hours. After 4 days, cells were passaged and cultured on mitotically inactivated mouse embryonic fibroblasts (MEFs). iPSC colonies were manually picked and passaged. Undifferentiated iPSCs and human embryonic stem cells (hESCs; H7 line; WiCell Research Institute, Madison, WI) were cultured and passaged on a MEF feeder layer with daily medium changes (DMEM/ F12, 20\% knockout serum replacement, $1 \mathrm{mM}$ L-glutamine, $0.1 \mathrm{mM}$ nonessential amino acids, $0.1 \mathrm{mM} \beta$-mercaptoethanol [Sigma], and $4 \mathrm{ng} / \mathrm{ml}$ human basic fibroblast growth factor [bFGF; from Invitrogen except as noted]) in dedicated incubators $\left(37^{\circ} \mathrm{C}, 5 \% \mathrm{CO}_{2}\right)$. For teratoma assays, DS and control iPSCs were cultured on MatriGel-coated dishes (BD Biosciences, Franklin Lakes, NJ) in MEF-conditioned media. Cells were dissociated with trypsin, suspended in phosphate-buffered saline $\left(1 \times 10^{7} \mathrm{cells} / \mathrm{ml}\right)$, and subcutaneously injected $(200 \mu \mathrm{l})$ in the upper hindlimbs of NOD SCID gamma mice (Jackson Laboratory, Bar Harbor, ME). After 6 to 8 weeks, tumors were dissected and fixed with $4 \%$ paraformaldehyde. Tissues were embedded in OCT, frozen, sectioned, and stained with hematoxylin and eosin. To generate neural progenitor cells (NPCs), iPSCs were differentiated via embryoid bodies (EBs) for 4 to 5 days and plated on Matrigel-coated dishes in NPC medium (DMEM/F12, N-2 supplement, 20ng/ml bFGF, nonessential amino acids, $2 \mathrm{mM}$ glutamine, and $2 \mu \mathrm{g} / \mathrm{ml}$ heparin [Sigma]). After 3 weeks, neural rosettes were isolated, and NPCs were expanded as floating neurospheres. For neuronal differentiation, neurospheres were dissociated with trypsin, and cells plated on Matrigel-coated dishes were cultured in differentiation medium lacking mitogens (Neurobasal plus B27 [Invitrogen], 10ng/ml brain-derived neurotrophic factor and neurotrophin-3 [PeproTech, Rocky Hill, NJ]) for 2 months.

\section{Reverse Transcriptase Polymerase Chain Reaction and Gene Expression Profiling}

Total RNA was extracted from iPSCs, hESCs, fibroblasts, and neural progeny with the RNeasy Mini or Micro kit (Qiagen, Hilden, Germany) and DNAse I treatment. First-strand cDNA synthesis was done from $2 \mu \mathrm{g}$ of total RNA using Superscript III reverse transcriptase (Invitrogen) and random hexamers according to the manufacturer's instructions. Polymerase chain reaction (PCR) was performed in $25 \mu \mathrm{l}$ reactions containing $200 \mathrm{nM}$ each of forward and reverse primers, $1 \times$ PCR buffer, Taq DNA polymerase, and cDNA template. PCR primer sequence and annealing temperature are provided in the Supplementary Table. For reverse transcriptase (RT)-PCR of sodium channel genes, cDNA was synthesized from 250ng of total RNA using the Superscript First Strand cDNA Synthesis Kit (Invitrogen). PCR was performed in $25 \mu \mathrm{l}$ reactions containing $500 \mathrm{nM}$ each of forward and reverse primers, $1 \times \mathrm{PCR}$ buffer, 200nM deoxyribonucleoside triphosphate, 1U Taq DNA polymerase (Promega, Madison, WI), and cDNA template. Amplification was carried out with an initial 3-minute denaturation at $94^{\circ} \mathrm{C}$, followed by 35 cycles of 45 seconds at $94^{\circ} \mathrm{C}$, 45 seconds at $60^{\circ} \mathrm{C}$, and 45 seconds at $72^{\circ} \mathrm{C}$, and terminating with a 10 -minute extension reaction at $72^{\circ} \mathrm{C}$. The forward primer $S C N 1 A \times 13$ is located in exon 13, and the reverse primer $S C N 1 A \mathrm{x} 15$ is located in exon 15; this primer pair amplifies a 343 base pair (bp) fragment from the wild-type transcript and a 169-bp fragment from the DS1 transcript lacking exon 14 (174 bp in length). To obtain gene-specific amplification of sodium channel transcripts, primer pairs were designed with the $3^{\prime}$ terminus of 1 or both primers terminating on 1 to 2 bases that are specific to 1 gene in the sodium channel family (see Supplementary Table). Sodium channel primers designated $\mathrm{F}$ and $\mathrm{R}$ are located in the penultimate and final exon of each gene, respectively. Amplified fragments were sequenced to confirm specificity. Quantitative RT-PCR for exogenous Oct4, Klf4, Sox2, and c-Myc was performed with the SYBR Green qPCR reagent (Applied Biosystems, Foster City, CA) containing $200 \mathrm{nM}$ each of forward and reverse primers and template using a Bio-Rad (Hercules, CA) iCycler PCR machine. For microarrays, total RNA (400ng) was labeled with the Total Prep RNA amplification kit (Ambion), and biotin-labeled complementary RNA (750ng) was prepared for hybridization to the Illumina (San Diego, CA) HumanHT-12 v4 Expression BeadChip. Chips were washed, labeled with $\mathrm{Cy} 3$, scanned into an Illumina BeadArray Reader, and analyzed with GenomeStudio (Illumina).

\section{Immunocytochemistry and Microscopy}

Cultures were fixed with $4 \%$ paraformaldehyde for immunocytochemistry. For immunostaining involving nuclear and cytoplasmic proteins, cells were permeabilized with $0.2 \%$ Triton X-100. Antibodies used were: Pax6 (1:100; Developmental 
Studies Hybridoma Bank, University of Iowa)), Musashi (1:500; Chemicon, Temecula, CA), FORSE-1 (1:1,000; Chemicon), Otx2 (1:500; R\&D Systems, Minneapolis, MN), En1 (1:500; Chemicon), Oct3/4 (1:200; Santa Cruz Biotechnology, Santa Cruz, CA), Nanog (1:500; Chemicon), SSEA-4 (1:200; Chemicon), SMA (1:1,000; Sigma), $\alpha$-fetoprotein (1:500; Dako, Carpinteria, CA), $\beta$-III-tubulin (TuJ1 clone, 1:2,000; Covance, Emeryville, CA), microtubule-associated protein (MAP) 2ab (1:1,000; Sigma), Tbr-1 (1:400; Abcam, Cambridge, MA), $\gamma$-aminobutyric acid (GABA; 1:3,000; Sigma), vesicular glutamate transporter-1 (VGLUT1; 1:4,000; Chemicon), parvalbumin (1:400; Abcam), calretinin (1:2,000; Chemicon), calbindin (1:500; Abcam), somatostatin (1:300; Millipore), tyrosine hydroxylase (1:400; Chemicon), vimentin (1:1,000; MBL International, Woburn, MA), GFAP (1:1,000, Sigma), and S-100 $\beta$ (1:400; Swant, Marly, Switzerland). Conjugated secondary antibodies were applied, and nuclei were counterstained with bisbenzimide. Stained cells were viewed and imaged using epifluorescence microscopy (DM IRB; Leica, Wetzlar, Germany) and a SPOT RT digital camera (SPOT Imaging Solutions, Sterling Heights, MI).

\section{Statistical Analysis}

Voltage clamp data were tested for normality using the Shapiro-Wilk test. Continuous variables with normal distribution were compared using 2-tailed Student $t$ test or analysis of variance. Current clamp data from patient and control iPSCs were compared using Student $t$ test. Values were considered statistically significant at $p \leq 0.05$.

\section{Results}

A Novel Loss-of-Function SCN1A Splice Site Mutation in a 7-Year-Old Patient with DS

The first subject's seizures began at age 9 months, his development subsequently regressed, and he experienced multiple types of pharmacoresistant seizures. Diagnostic sequencing identified a heterozygous splice donor site mutation IVS14+3A $>$ T (Fig 1A). This mutation substitutes a pyrimidine at the important +3 position that is occupied by a purine ( $\mathrm{G}$ or $\mathrm{A}$ ) at $>95 \%$ of human splice sites. The subject's parents do not carry the mutation (data not shown), consistent with a de novo pathogenic mutation, as is frequently seen in DS. ${ }^{6}$ RT-PCR of RNA from the cultured fibroblasts using primers in exon 13 and exon 15 yields 2 major products, the product of the wild-type allele that contains exon 14 and the mutant product that skips exon 14 (see Fig 1B). The identity of RT-PCR products was confirmed by sequencing. Using primers in exon 11 and exon 15, a less abundant transcript that skips both exons 13 and 14 was detected (data not shown). Because exon 14 contains $174 \mathrm{bp}$, the open reading frame is maintained in the mutant transcript, but the predicted protein product has deletion of transmembrane segments 2 and 3 in domain 2, with predicted loss of channel function (see Fig 1C).

To test the function of the mutant channel, we expressed either wild-type human $S C N 1 A$ cDNA or a cDNA lacking the codons from exon 14 in Xenopus oocytes and recorded whole-cell sodium currents under voltage clamp. Consistent with previous findings, ${ }^{26}$ injection of RNA encoding wild-type human SCN1A resulted in the expected whole-cell sodium currents (see Fig 1Di). In contrast, injection of the mutant RNA failed to produce any detectable current (see Fig 1Dii). We next transfected mammalian tsa-201 cells with cDNA encoding wild-type or mutant SCN1A and performed immunoblots of cell lysates. Robust expression of $\mathrm{Na}_{\mathrm{v}} 1.1$ channel protein was obtained with wild-type SCN1A, but the mutant $\mathrm{Na}_{\mathrm{v}} 1.1$ protein was not detectable above background (see Fig 1E, F), although we cannot exclude expression of a markedly truncated, nonfunctional protein. Together, these findings demonstrate that the mutant protein is unstable and does not produce functional channels at the cell membrane. We also expressed mutant and wild-type channel alone or in combination in tsa-201 cells and found no current expressed by mutant channel alone, and no influence of the mutant channel on wild-type current to suggest a dominant-negative effect (data not shown). Thus, this patient is heterozygous for a null allele of SCN1A, as characteristic for DS. ${ }^{6}$

\section{Generation of DS Patient and Control iPSCs and Neurons}

Heterologous expression systems provide only limited data regarding the actual function of a mutant channel in the cells of physiological interest, which in the case of epilepsy are forebrain neurons. Moreover, differences in genetic background both between and within species may substantially influence neuronal ion channel function. ${ }^{27}$ To characterize the net in vivo effect of the SCN1A mutation in the genomic background of genetic modifiers, we chose to study the functional effects of DS patient SCN1A mutations using the subjects' own neurons via the iPSC method. Primary fibroblasts were derived from skin biopsies obtained from 2 DS subjects (both aged 7 years) and 3 unaffected controls (aged 1, 10 , and 25 years), and were reprogrammed by retroviralmediated gene transfer of Oct4, Sox2, Klf4, and cMyc. ${ }^{14,15}$ We initially worked with fibroblasts from the DS patient carrying the IVS $14+3 \mathrm{~A}>\mathrm{T}$ mutation described above, and the majority of our results are from this subject (DS1). We subsequently reprogrammed cultured fibroblasts from an additional subject with DS (DS2) who has a c.975T $>$ A SCN1A nonsense mutation in tyrosine codon 325, resulting in the premature 

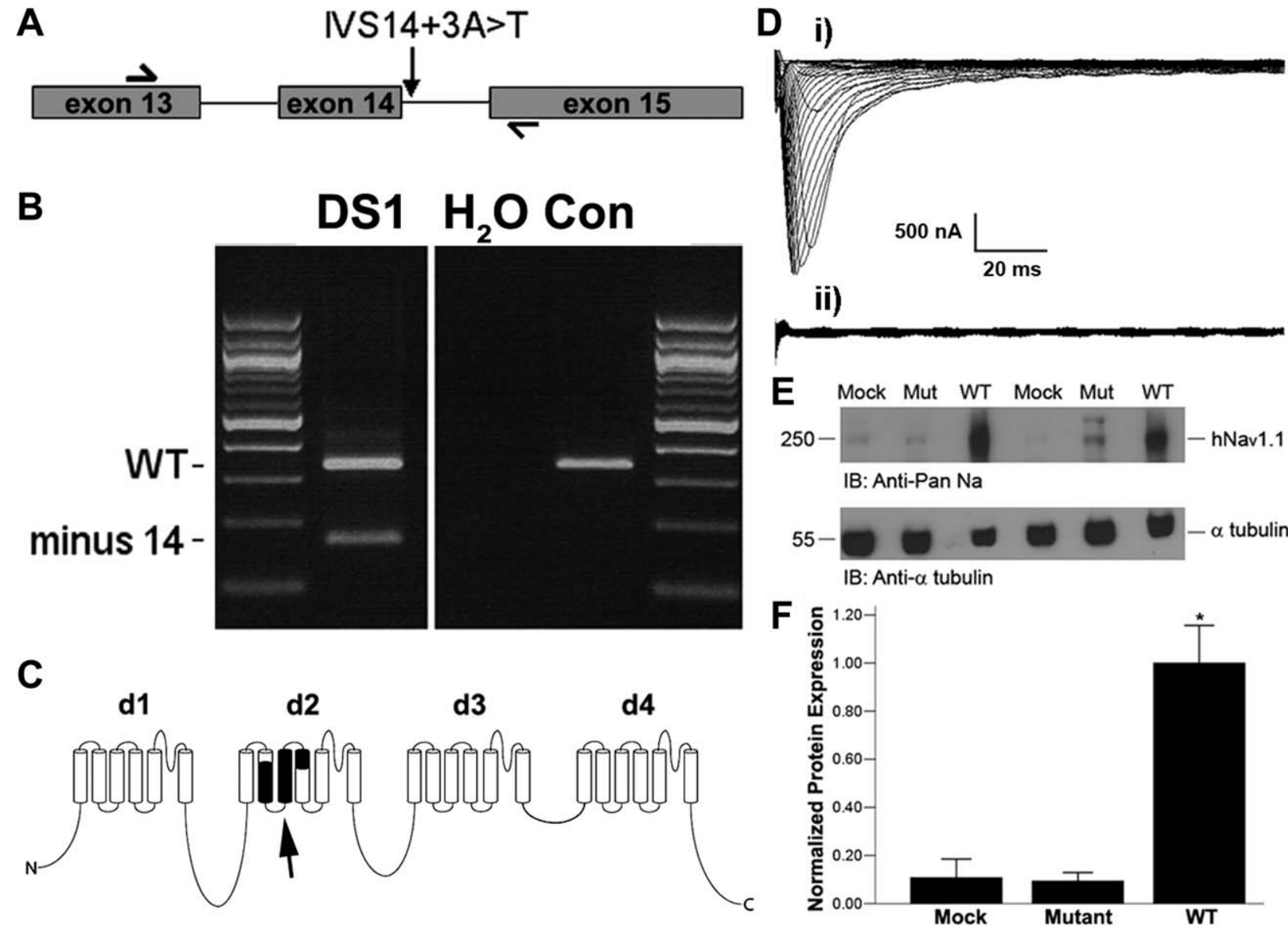

FIGURE 1: SCN1A transcripts in fibroblast cultures from patient DS1 (splice donor site mutation IVS14+3 A $>T$ in intron 14). (A) The mutation is located $3 \mathrm{bp}$ downstream of exon 14. Polymerase chain reaction (PCR) primers are located in exons 13 and 15. (B) Reverse transcriptase PCR products from a Dravet syndrome (DS) patient and control (CON) fibroblasts. In addition to the wild-type (WT) product of $340 \mathrm{bp}$ that contains exon 14, the patient's fibroblasts produce a smaller product that skips exon 14, as confirmed by sequencing. (C) Diagram of $\mathrm{Na}_{\mathrm{v}} 1.1$ channel protein $\alpha$ subunit, with exon 14 residues in black (arrow). (D) Traces of 2 electrode voltage clamp recordings from Xenopus oocytes injected with RNA of human WT (i) or IVS14+3A>T DS mutant (ii) SCN1A. Only the WT generates sodium current. Scale bar: vertical $=500 \mathrm{nA}$; horizontal $=20 \mathrm{milliseconds}$. (E) $\mathrm{Im}$ munoblots (IBs) of tsa-201 cells transfected with WT or IVS14+3A>T mutant (Mut) SCN1A using anti-Nav1.1 antibody. WT shows strong bands (2 replicates), whereas the mutant shows little or no labeling above the mock-transfected control. $\alpha$-Tubulin is the loading control. (F) Densitometric quantification of $\mathrm{Na}_{\mathrm{v}} 1.1$ immunoblots from several separate cultures normalized to $\alpha$-tubulin expression. ${ }^{*} p<0.001$, analysis of variance with Tamhane T2 as post hoc test.

termination codon TAA (Y325X). This mutation truncates the 4-domain sodium channel protein within the pore loop of domain 1, resulting in a predicted loss of channel activity. A de novo missense mutation in the same codon, Y325C, was previously observed in a patient with DS (SCN1A Variant Database, http://www.molgen.vib-ua.be/SCN1AMutations).

Colonies with hESC-like morphology emerged within 10 to 14 days after fibroblast reprogramming, and by 30 days they displayed the morphology of pluripotent stem cells similar to a simultaneously cultured H7 hESC line (Fig 2 A-D). At 3 to 4 weeks after reprogramming, colonies were manually picked and individually passaged onto irradiated MEF feeder cells. RT-PCR analysis of SCN1A showed that the mutant transcript lacking exon 14 was expressed in DS1 patient-derived iPSCs
(Supplementary Fig 1). Consistent with successful reprogramming into iPSCs, cells from 4 lines (from DS1, DS2, and 2 controls) were injected into immunocompromised mice and formed teratomas (see Supplementary Fig 1B and data not shown). Reprogrammed iPSCs lost expression of a fibroblast-associated antigen, TE-7 (data not shown), and all iPSC clones from DS patients and controls, unlike the fibroblasts, continuously expressed pluripotency markers, including Nanog, Oct3/ 4, SSEA4, alkaline phosphatase, and others. We also compared the global gene expression profiles among undifferentiated patient and control iPSCs, H7 cells, and fibroblasts that were not reprogrammed. As expected, the iPSCs showed a gene expression profile that was much more similar to $\mathrm{H} 7$ hESCs than to the original fibroblasts from which they were derived. RT-PCR for the 

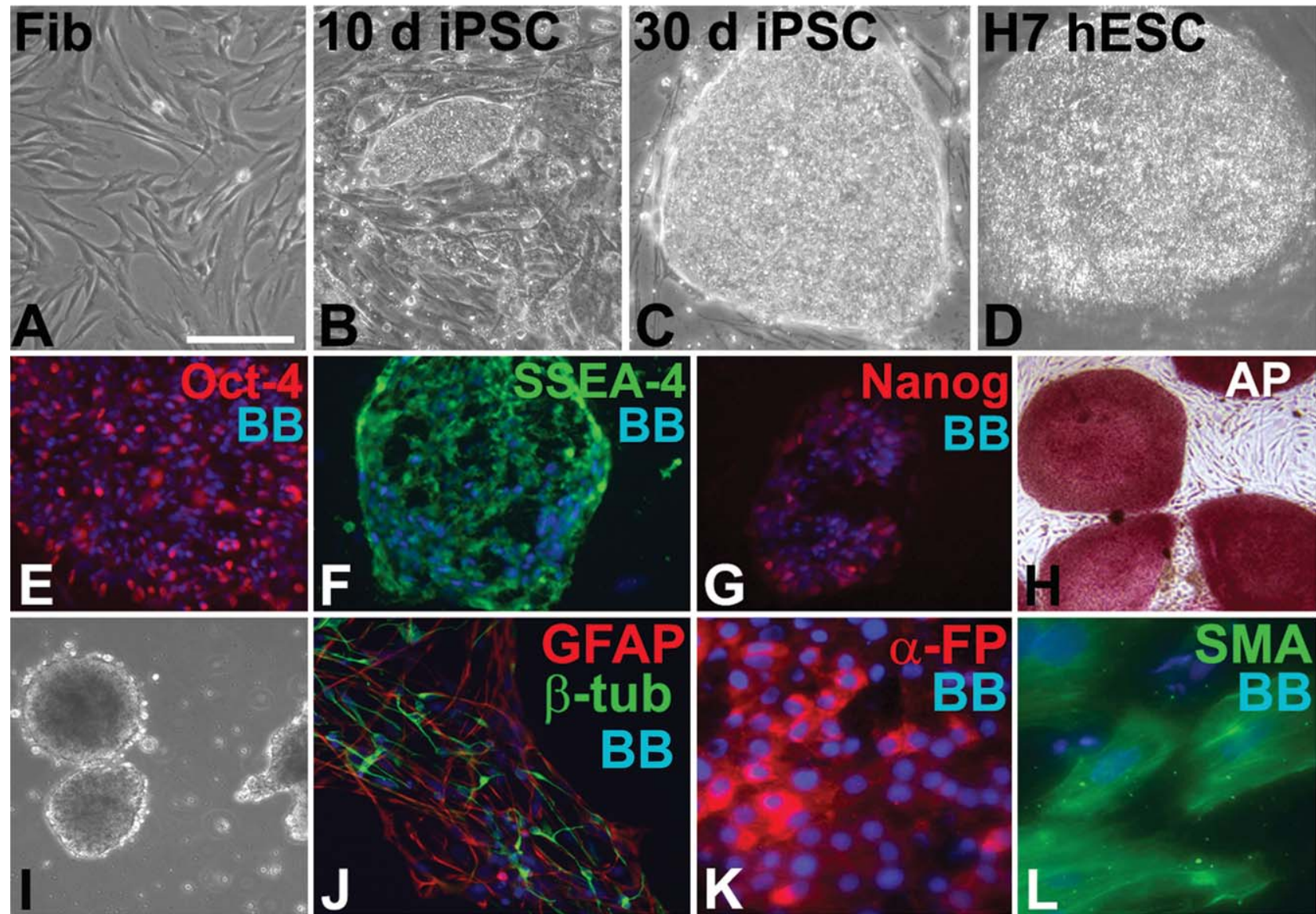

FIGURE 2: Reprogramming Dravet syndrome (DS) patient fibroblasts into induced pluripotent stem cells (iPSCs). (A) Skin biopsy-derived fibroblasts from patient DS1 before reprogramming. (B-D) After 4-factor retroviral reprogramming, pluripotent stem cell colonies began to form within 10 days (B), and were well developed by 30 days (C), and their morphology was similar to a human embryonic stem cell (hESC) colony from the H7 line (D). (E-G) Patient-specific iPSCs expressed pluripotency markers Oct4 (E), stage-specific embryonic antigen-4 (SSEA-4; F), Nanog (G), and alkaline phosphatase (AP; H). (I-L) Differentiation of patient-specific iPSCs through embryoid bodies (I) led to tissue of ectodermal (J), endodermal (K), and mesodermal (L) lineages. $\beta$-tub $=$ neuron-specific $\beta$-tubulin; GFAP $=$ glial fibrillary acidic protein; $\alpha$-FP $=\alpha$-fetoprotein; SMA $=$ smooth muscle actin. Bisbenzimide (BB) nuclear stain is blue in E-G and J-L. Scale bar $=100 \mu \mathrm{m}$ for A-G, $200 \mu \mathrm{m}$ for H-J, $50 \mu \mathrm{m}$ for K, L. [Color figure can be viewed in the online issue, which is available at www.annalsofneurology.org.]

exogenous transcription factor transgenes used for reprogramming showed marked downregulation after fibroblasts were converted to iPSCs. Also consistent with a pluripotent cell phenotype, control and patient-derived iPSCs could be differentiated through EBs to give rise to cells comprising all 3 embryonic germ layers (see Fig 2I-L and data not shown).

Because forebrain neurons are the affected cells that cause disease in DS, we directed the differentiation of patient and control iPSCs toward a forebrain NPC phenotype. Five-day-old EBs were cultured to generate NPCs. Early NPCs displayed a neural rosette morphology and expressed forebrain-specific regionalization markers but not more caudal central nervous system markers or a pluripotency gene (Fig 3; Supplementary Fig 2). Neural rosettes were manually picked, passaged, dissociated, and differentiated to generate neurons and glia. Early differentiating cells (2-7 weeks) expressed neuronal antigens, including neuron-specific $\beta$-tubulin and MAP2 (Supplementary Fig 3B-D), and exhibited increased expression of 5 voltage-gated sodium channels predominantly expressed in neurons (see Supplementary Fig 3A). Approximately 80 to $90 \%$ of the neurons displayed a bipolar morphology and expression of GABA characteristic of an interneuron phenotype, whereas about $10 \%$ were pyramidal-shaped and expressed the VGLUT1 and the forebrain pyramidal cell marker Tbr1 (see Fig 2 and Supplementary Fig 2E). Rare cells expressed tyrosine hydroxylase, indicative of a monoaminergic cell type. To determine the types of interneurons generated from the iPSCs, we immunostained for different forebrain interneuron subtypes and found subsets of cells expressing calretinin, calbindin, parvalbumin, or somatostatin (see Supplementary Fig 3B-D and data not shown). A predominance of interneurons generated by pluripotent stem cells is not unusual, ${ }^{28-30}$ although other 

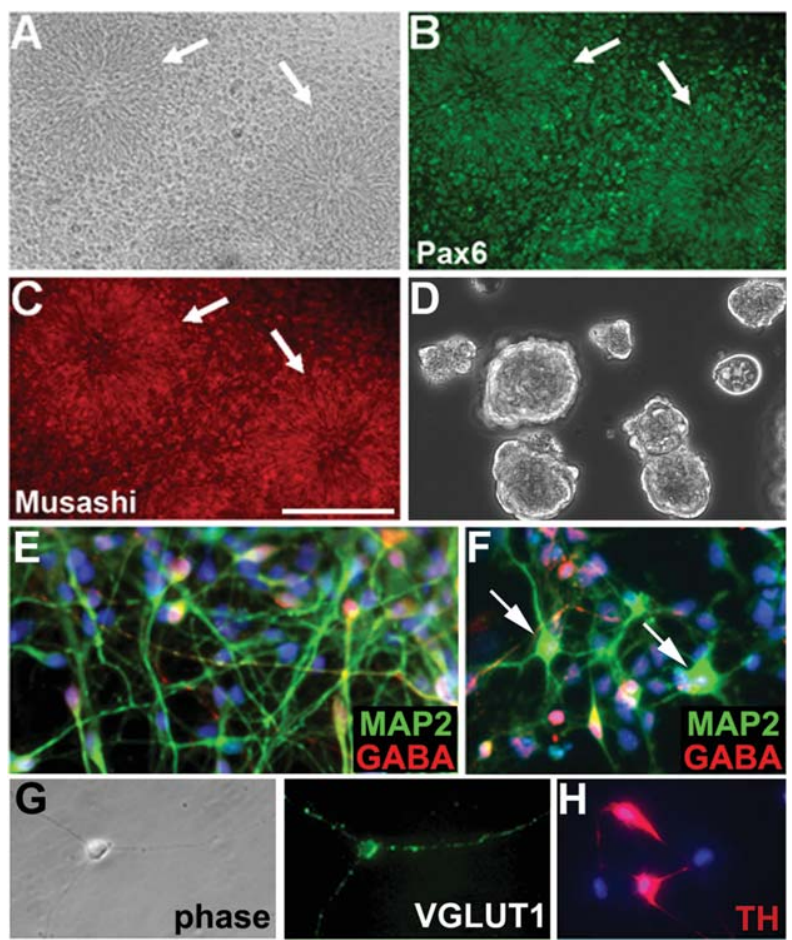

FIGURE 3: Neuronal differentiation of Dravet syndrome patient-specific induced pluripotent stem cells (iPSCs). (A-D) Mutant iPSCs were differentiated into neural rosettes (arrows in $\mathrm{A}-\mathrm{C}$ ) that expressed the neural progenitor cell (NPC) markers Pax6 (B) and Musashi (C), and then were expanded as neurospheres (D). (E-G) Differentiation of iPSC-derived NPCs into microtubule-associated protein (MAP) $2^{+}$(green in E, F) induced neurons yielded mostly $\gamma$ aminobutyric acidergic (GABAergic) interneurons (red in $E$, $\mathrm{F}$ ), and about $10 \%$ pyramidal shaped neurons (arrows in F) that expressed the vesicular glutamate transporter-1 (VGLUT1) (G); phase image is shown in the left-hand panel of (G). (H) Rare cells expressed tyrosine hydroxylase (TH). Bisbenzimide nuclear stain is blue in $E, F, H$. Scale bar $=200 \mu \mathrm{m}$ for A-C, $100 \mu \mathrm{m}$ for $\mathrm{D}, 75 \mu \mathrm{m}$ for $\mathrm{E}-\mathrm{H}$. [Color figure can be viewed in the online issue, which is available at www.annalsofneurology.org.]

culture conditions produce more pyramidal neurons (eg, Shi et $\mathrm{al}^{31}$ ). With longer culture durations (7-12 weeks), there appeared substantial numbers of glia that expressed glial fibrillary acidic protein, $S 100 \beta$, and vimentin (data not shown). Although neuronal RNA splicing may vary from that of other cell types, we found that iPSC-derived neurons (herein called induced neurons) from subject DS1 with the IVS14 $+3 \mathrm{~A}>\mathrm{T}$ mutation retained the abnormal exon 14 splicing of SCN1A described above for fibroblasts and iPSCs (see Supplementary Fig 1A).

\section{Increased Sodium Current Density and} Neuronal Excitability in SCN1A Mutant Neurons To examine whether induced neurons from DS subjects and controls differed in ways that may predispose to epileptic seizures, we performed whole-cell voltage and current clamp recordings. We first recorded sodium currents and measured current densities under voltage clamp in induced neurons that were differentiated for 5 to 7 days. As expected, given the upregulation of voltagegated sodium channel mRNAs (see Supplementary Fig $3 \mathrm{~A})$, both bipolar- and pyramidal-shaped neurons from DS1 (mutant) and controls showed typical sodium currents (Supplementary Fig 4). No significant differences in sodium current densities were observed between DS1 and control induced neurons at this time point, or between bipolar- and pyramidal-shaped neurons within groups (see Supplementary Fig 4B).

We next measured sodium current densities of induced neurons after differentiation for 3 to 5 weeks. Neurons with both pyramidal- and bipolar-shaped morphologies from DS subjects and controls showed sodium currents that were blocked with the addition of $300 \mathrm{nM}$ tetrodotoxin (TTX; Fig 4; Supplementary Fig 4C), a finding consistent with neuronal TTX-sensitive sodium currents. However, we observed significantly increased (2-fold) mean sodium current densities in 3- to 5-weekdifferentiated DS mutant neurons with both pyramidal and bipolar morphologies derived from DS1 compared to controls. Induced mutant neurons of both morphologies derived from DS2 exhibited 3-fold increases in sodium current density compared to controls. Sodium current densities in control neurons remained similar to the levels measured at 5 to 7 days. No significant differences in sodium current densities were present between bipolar- and pyramidal-shaped neurons within groups. In contrast to current density, the voltage-dependence and kinetics of current activation and inactivation were not different between control and mutant induced neurons (see Supplementary Fig 4D-E).

To examine whether the increase in sodium current density was associated with altered neuronal excitability of mutant neurons, we recorded action potentials from DS mutant and control induced neurons differentiated for 3 to 7 weeks under current clamp recording conditions. In both mutant and control neurons, action potentials were evoked with stimulation after 4 weeks of differentiation, with more consistently produced repetitive firing by 5 weeks. DS1- or DS2-derived mutant induced bipolar and pyramidal neurons differentiated for 5 to 7 weeks exhibited a significantly reduced threshold for action potential generation and increased repetitive firing frequency compared to controls (Fig 5; Table 1). Moreover, mutant pyramidal- and bipolar-shaped neurons frequently displayed spontaneous repetitive firing and bursting activity that was almost never observed in control neurons (Supplementary Fig 5; Table 2). Changes in neuronal excitability in mutant pyramidal and bipolar 
A (i)

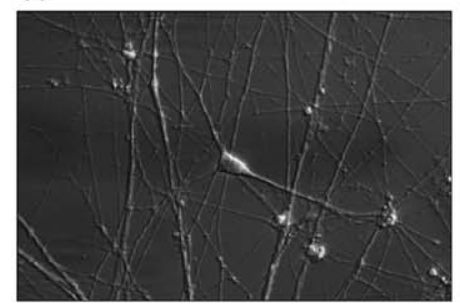

B

(i)

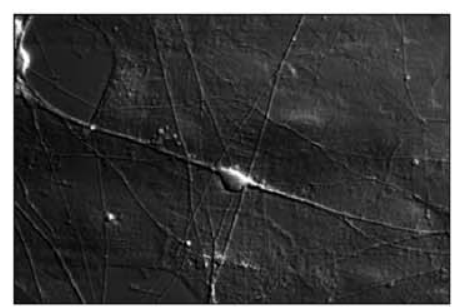

Pyramidal

(ii)

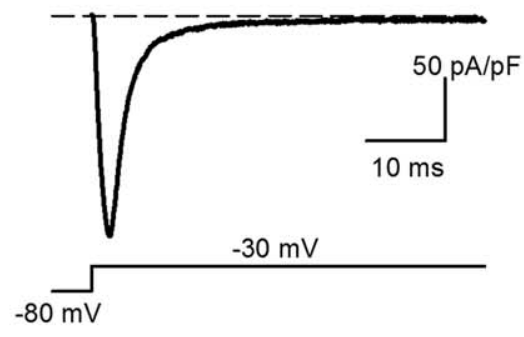

Bipolar
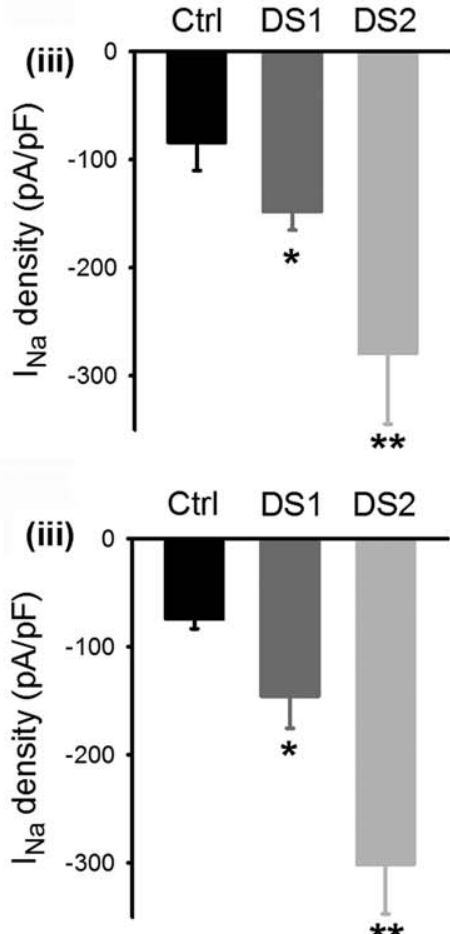

FIGURE 4: Sodium current $\left(I_{\mathrm{Na}}\right)$ density is increased in pyramidal and bipolar neurons with SCN1A mutations. (A, B) Induced neurons with pyramidal (A) or bipolar (B) morphology are shown in phase images on the left (i). Representative traces of $I_{\mathrm{Na}}$ (ii) recorded using the protocol displayed in the inset are shown, as are bar graphs of the mean $I_{\mathrm{Na}}$ density from control (Ctrl) and DS1 and DS2 mutant pyramidal neurons (Aiii; control: black bars, $n=14$; DS1: dark gray bars, $n=22, p=0.012$ vs control; DS2: light gray bars, $n=6, p=0.023$ vs control) and bipolar neurons (Biii; control, $n=25 ; D S 1, n=27, p=0.048$ vs control; DS2, $n=5, p=0.001$ vs control). ${ }^{\star} p<0.05 ;{ }^{*} p<0.01$. No significant differences in sodium current densities are present between bipolar and pyramidal neurons within groups ( $p=0.70$ and 0.94 for control and DS1, respectively). Error bars indicate mean \pm standard error of the mean.

neurons from DS1 and DS2 were virtually identical, and pooled data for the 2 subjects are shown in Tables 1 and 2. Thus, both loss-of-function mutations in SCN1A increased neuronal excitability. To confirm that sodium current densities remained increased in DS induced neurons after 5 to 7 weeks of differentiation, we measured current densities under voltage clamp in these more mature neurons. Sodium current densities in bipolarand pyramidal-shaped DS1 induced neurons differentiated for 5 to 7 weeks remained significantly increased compared to control induced neurons of the same maturity (Supplementary Fig 6), and no significant differences were present between bipolar- and pyramidal-shaped neurons within groups.

Consistent with the increased sodium current densities shown in Figure 4 and Supplementary Figure 6, the mutant neurons also displayed more depolarized resting membrane potentials than those of the control neurons, although the difference was only significant for bipolarshaped cells (see Table 1). However, there were no differences in other intrinsic membrane electrical properties, including membrane capacitance and input resistance, between DS and control induced neurons. All of the current and voltage clamp recordings were consistent for 3 sublines from DS1, 2 sublines from DS2, and all 3 controls. Taken together, these data suggest that pyramidaland bipolar-shaped DS mutant induced neurons are hyperexcitable, a result consistent with an in vitro epileptic-like phenotype.

\section{Quantitation of Sodium Channel Transcripts}

The level of sodium channel transcripts increases during the transition from iPSCs to derived neurons (see Supplementary Fig 3A). To determine whether there was transcriptional upregulation of 1 or more sodium channel genes in induced neurons from DS patients compared to controls, we carried out RT-PCR using gene-specific primers for the 5 sodium channel genes that are expressed in these cells: SCN1A, SCN2A, SCN3A, SCN8A, and SCN9A. None of the sodium channel transcripts were elevated in patientderived neurons compared with control neurons (Fig 6). These data indicate that the elevated sodium current density in patient-derived neurons results from a post-transcriptional mechanism. 

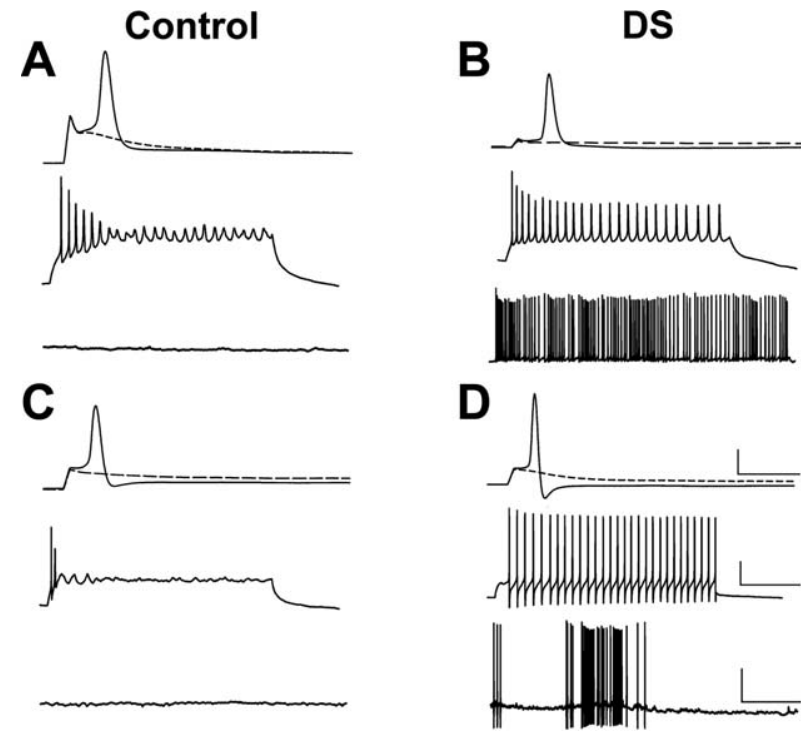

FIGURE 5: Characteristics of evoked action potentials (APs) and spontaneous firing in human induced neurons from control and Dravet syndrome (DS) subjects. Top trace in A-D shows representative APs (solid lines) recorded from control (A) or DS2 (B) bipolar-shaped cells or control (C) or DS1 (D) pyramidal-shaped cells cultured for 5 weeks using wholecell patch clamp recording in current clamp mode. APs evoked by injection of threshold depolarizing currents required much less current for the DS2 bipolar $(B ; 0.17 n A, 1$ millisecond) or DS1 pyramidal ( $D ; 0.27 n A, 1$ millisecond) induced neurons than for the control bipolar $(A ; 0.61 \mathrm{nA}, 1$ millisecond) or pyramidal ( $C ; 0.35 \mathrm{nA}, 1$ millisecond) induced neurons; cells were held at resting membrane potential $(-65 \mathrm{mV}$ for the control bipolar, $-63 \mathrm{mV}$ for control pyramidal cells, $-55 \mathrm{mV}$ for DS2 bipolar, and $-72 \mathrm{mV}$ for DS1 pyramidal cells, respectively). Also shown are the responses evoked by subthreshold depolarizing current injections in the same cells (dotted lines of the top traces in A-D). Middle traces in A-D represent repetitive firing evoked by injection of a long-pulse depolarizing current $(0.02 \mathrm{nA}, 1,500$ milliseconds) in the same cells as in the top traces. Note the higher frequency and more prolonged firing in the DS1 or DS2 induced neurons $(B, D)$ than in the controls $(A, C)$. Bottom traces in A-D show that no spontaneous firing was observed in either control bipolar (A) or pyramidal (C) induced neurons when they were held at resting membrane potential, but both DS1 and DS2 patient-specific induced neurons displayed consistent, spontaneous firing with bursting activity at resting membrane potential $(B, D)$. Each trace is a representative example of 7 to 45 individual cells. Scale bars $=40 \mathrm{pA}$ for all vertical bars; 10 milliseconds, 400 milliseconds, and 10 seconds for horizontal bars of the top, middle, and bottom traces of each panel, respectively.

\section{Discussion}

We used the iPSC method to compare the physiological properties and sodium channel subunit expression of forebrainlike neurons from 2 DS subjects and 3 human controls. Voltage clamp recordings initially (5-7 days) showed sodium current densities that were not significantly different between induced neurons from DS subjects and controls. The neurons were very immature and lacked action potentials at this early stage; however, with further maturation in vitro to functional neurons, DS induced neurons developed significantly greater sodium current densities than those of controls. These findings were present in both putative excitatory pyramidalshaped neurons and inhibitory neurons with bipolar morphology. Current clamp recordings showed that DS induced neurons of both morphologies were hyperexcitable compared to controls, with significantly reduced thresholds for action potential generation and increased firing frequency, as well as spontaneous repetitive firing and bursting behavior. These findings indicate that DS iPSC-derived neurons model an epileptic-like phenotype with in vitro seizure-like activity.

The functional effects of SCN1A mutations were initially explored using voltage clamp recordings in heterologous expression systems such as Xenopus oocytes or nonneuronal mammalian cell lines. ${ }^{10,11}$ An inconsistent mixture of loss-of-function and gain-of-function effects of individual mutations was obtained with this approach. ${ }^{6,9,32}$ The advantages of the iPSC approach over these heterologous expression systems are underscored in the present study. The mutant channels are present in the natural host cells without the need for transfection. In addition, the patient genetic background is maintained, and therefore the properties of the induced neurons reflect the net effect of the mutation and genetic modifiers.

Mouse models of DS have been generated by targeted deletion of mouse Scnla and knock-in of a human SCN1A premature truncation mutation causing DS. ${ }^{12,13}$ Homozygous and heterozygous Scnla null mice display spontaneous seizures; the homozygotes are ataxic and die at the beginning of the third postnatal week, whereas more than half of the heterozygotes survive to adulthood. ${ }^{12}$ Voltage clamp recordings from acutely dissociated hippocampal neurons derived from heterozygous and homozygous Scnla null mice revealed decreased sodium current densities in putative interneurons with bipolar morphology, but not in neurons with pyramidal morphology. ${ }^{12,13}$ The interneurons also showed decreased current-induced repetitive firing and reduced action potential amplitudes. A compensatory upregulation of $\mathrm{Na}_{\mathrm{v}} 1.3$ was seen in homozygous and heterozygous Scnla null mice, but this was not sufficient to restore normal sodium currents in putative interneurons. Similarly, studies using a knock-in mouse model of DS revealed decreased bursting of fast-spiking interneurons in in vitro cortical slices from heterozygotes. ${ }^{13}$ Together, findings from these mouse DS models, along with a more recent study using mice with conditional deletion of Scnla in interneuron progenitors, ${ }^{33}$ led to the interneuron hypothesis of epileptogenesis in DS, whereby decreased 
TABLE 1. Comparisons of Passive and Evoked Membrane Electrical Properties of Bipolar- and PyramidalShaped Induced Neurons between Control and DS Patients

\begin{tabular}{lllllll} 
Group & Resting $\mathbf{V}_{\mathbf{m}}, \mathbf{m} \mathbf{V}$ & \multicolumn{2}{l}{ Input Resistance, $\mathbf{G} \boldsymbol{\Omega}$} & $\mathbf{V}_{\text {Threshold, }} \mathbf{m V}$ & AP Amplitude, $\mathbf{m V}$ & AP Frequency, $\mathbf{H z}$ \\
B-Ctrl & $-67.15 \pm 1.37(33)$ & $1.25 \pm 0.15(33)$ & $-23.67 \pm 1.51(30)$ & $114.90 \pm 4.69(34)$ & $3.48 \pm 0.73(27)$ \\
\hline B-DS & $-58.55 \pm 1.28(45)^{\mathrm{a}}$ & $1.24 \pm 0.12(45)$ & $-30.83 \pm 1.53(37)^{\mathrm{a}}$ & $121.18 \pm 3.26(37)$ & $11.6 \pm 1.33(37)^{\mathrm{a}}$ \\
P-Ctrl & $-63.05 \pm 2.72(9)$ & $1.02 \pm 0.34(9)$ & $-20.51 \pm 3.37(8)$ & $125.38 \pm 5.14(8)$ & $5.10 \pm 2.66(7)$ \\
\hline P-DS & $-58.08 \pm 1.24(31)$ & $1.02 \pm 0.17(31)$ & $-37.32 \pm 1.51(26)^{\mathrm{a}}$ & $114.35 \pm 3.97(26)$ & $12.64 \pm 1.99(24)^{\mathrm{b}}$
\end{tabular}

Values are mean \pm standard error of the mean; cell numbers are in parentheses.

${ }^{\mathrm{a}} p<0.001, \mathrm{~b} p<0.05$; Student $t$ test or Mann-Whitney rank sum test.

$\mathrm{AP}=$ action potential; AP Amplitude = spike height measured from the resting membrane potential level to the peak; AP

Frequency $=$ frequency of repetitive firing of a given iPSC in response to injection of a 1,500-millisecond depolarizing current pulse $(0.02 \mathrm{nA})$; B-Ctrl = bipolar-shaped neurons from control iPSCs; B-DS = bipolar-shaped neurons from DS iPSCs; DS = Dravet syndrome; $\mathrm{iPSC}=$ induced pluripotent stem cell; P-Ctrl = pyramidal-shaped neurons from control iPSCs; P-DS = pyramidalshaped neurons from DS iPSCs; Resting $\mathrm{Vm}=$ resting membrane potential; $\mathrm{V}_{\text {Threshold }}=$ threshold membrane potential for evoking an AP.

excitability of inhibitory interneurons leads to network hyperexcitability and seizures.

Our data from human mutant DS iPSC neurons differ substantially from those in the murine DS models and do not support the idea of an interneuron-specific decrease in excitability. We observed increased sodium current densities and hyperexcitability of both bipolarshaped putative inhibitory interneurons and putative pyramidal neurons, suggesting an alternative mechanism of seizure generation that is cell-autonomous and not dependent upon inhibitory innervation. Such a mechanism is supported by more recent data from a mouse Scnla heterozygous knockout DS model, in which sodium current density in pyramidal neurons is initially unchanged, but then significantly increases by the fourth week of life. $^{34}$

Our findings raise 2 critical questions. First, what is the cause of the increased sodium current density in DS patient-specific neurons? We found that DS and control sodium currents were similar in immature neurons, and that sodium currents increase in DS neurons as they mature. These data suggest a cell autonomous, homeostatic-like mechanism that overcompensates, perhaps by overexpression of a different voltage-gated sodium channel $\alpha$-subunit or a proexcitatory change in another ion channel. We did not observe increases in other voltagegated sodium channel $\alpha$ subunit expression at the mRNA level, suggesting that if there is any compensatory increase in these subunits, it would be through post-transcriptional mechanisms or altered subcellular targeting.

Second, how might the similar changes that we see in both interneurons and pyramidal cells cause epilepsy? The most plausible explanation is that increased excitability of both principal and inhibitory neurons leads to network hyperexcitability or synchronization sufficient to produce seizures and cognitive dysfunction. This effect might be analogous to the administration of a chemoconvulsant, such as kainic acid, that increases the activity of both inhibitory and excitatory neurons with the net outcome of seizure generation. Another possibility is that early hyperexcitability of neurons during embryonic development leads to abnormal neuronal integration and resultant network hyperexcitability. Some studies have found minor malformations of cortical development in DS, but others have not (reviewed in Guerrini et $\mathrm{al}^{35}$ ). Another issue relates to the clinical phenomena of worsening seizures in many DS subjects treated with sodium-channel blocking anticonvulsants such as lamotrigine. $^{36}$ This phenomenon is not easily explained by a mechanism involving compensatory increases in sodium currents, as proposed here. However, there is currently no direct evidence in any DS model system explaining this clinical finding, which is not seen in all DS subjects. Furthermore, the interneuron hypothesis put forward

\begin{tabular}{|c|c|c|}
\hline iPSCs & Control (\%) & DS patient (\%) \\
\hline Pyramidal-shaped & $0(0 / 7)$ & $68(19 / 28)$ \\
\hline Bipolar-shaped & $4(1 / 27)$ & $49(18 / 37)$ \\
\hline Overall & $3(1 / 34)$ & $57(37 / 65)$ \\
\hline
\end{tabular}




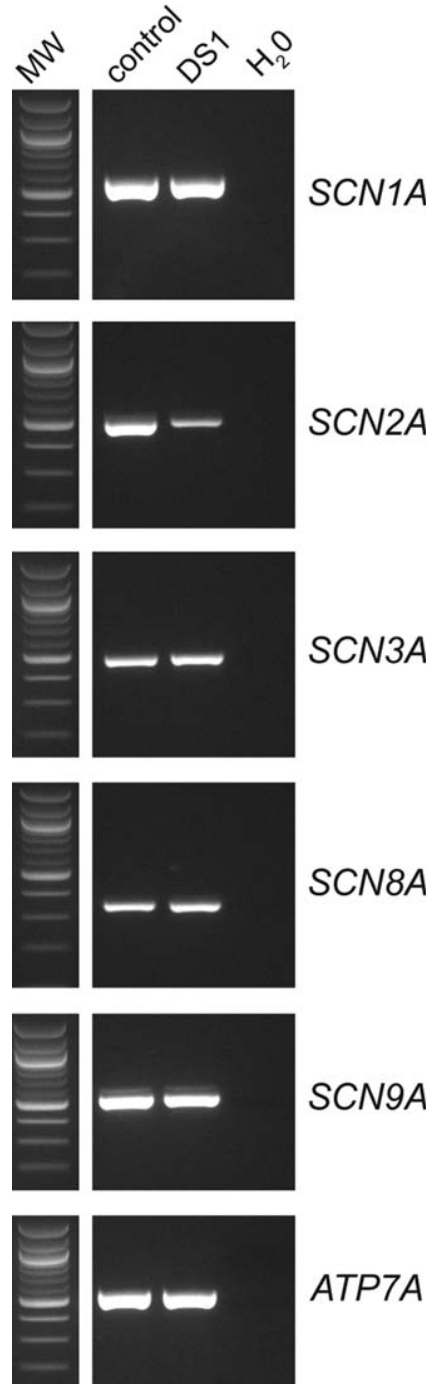

FIGURE 6: Sodium channel transcripts in induced pluripotent stem cell-derived neurons. Total RNA was isolated from induced neurons (5 weeks) from DS1 and control and amplified by reverse transcriptase polymerase chain reaction using gene-specific primers (see Supplementary Table) for 5 sodium channel genes and ATP7A (control), as indicated. Transcript abundance is not increased in the patient neurons.

based on the initial DS mouse model data also does not provide an adequate explanation. For example, the argument is made that if DS interneurons specifically lack $\mathrm{Na}_{\mathrm{v}} 1.1$, resulting in decreased sodium current DS, they would be affected to a greater extent than wild-type neurons by sodium channel blockers. However, one might just as well argue that pyramidal cells would be more affected in this situation, because they have higher levels of sodium current, more channels to block, and thus the drugs should work better. Additional caveats include the complicated network effects generated by other likely homeostatic mechanisms at play, and the often ignored issue that all of these anticonvulsants have multiple mechanisms of action.
Human iPSCs provide a remarkable experimental platform for understanding disease mechanisms and screening therapeutic drugs using patient-specific cells. For example, 3 recent studies reported the generation of patient-specific iPSCs and cardiac myocytes from subjects with 2 different forms of congenital long QT syndrome due to potassium channel gene mutations, ${ }^{18,19}$ and a third form of long QT syndrome due to a calcium channel mutation. ${ }^{20}$ The patient-specific cardiac myocytes modeled the disease phenotypes remarkably well, with action potential prolongation, increased in vitro arrhythmogenic potential, and responses to pharmacological agents that influence cardiac rhythmicity. Several patientspecific iPSC lines have been generated as models of other neurological diseases, ${ }^{17,37-39}$ but the mechanistic information derived thus far has been limited. A general limitation of the method is the possible impact of factors specific to the culture conditions or the state of differentiation of the iPSC-derived neurons. Nonetheless, our patient-specific DS model suggests that disease-causing neuronal ion channelopathies are amenable to modeling with iPSC-derived neurons. In addition to providing new insight into disease pathogenesis, the iPSC approach should prove an invaluable tool to evaluate candidate pharmacological agents using patient-specific cells.

\section{Acknowledgment}

This work was supported by grants from the NIH to M.H.M. (NINDS RC1NS068684), to L.L.I. (NINDS NS064245 and NINDS NS076752), and to Y.L. (NIMH MH059980), a grant from the American Epilepsy Society to J.M.P., a grant from the University of Michigan Rare Disease Initiative to L.L.I., a fellowship from the Epilepsy Foundation to G.A.P., a grant from the NIH (NHLBI T32HL007853) to the University of Michigan Cardiovascular Center for H.A.O., National Institute of General Medical Sciences Genetics Predoctoral Training Grant T32 GM007544 to J.E.O, and a grant from Istituto Neurologico C. Besta, Milan, Italy to R.R.

We thank K. S. O'Shea for generous assistance with the teratoma assays.

\section{Authorship}

Y.L., L.F.L.-S., Y.Y., M.H.M., L.L.I., and J.M.P. contributed equally.

\section{Potential Conflicts of Interest}

G.A.P.: grants/grants pending, NIH. A.G.: grants/grants pending, TS Alliance; speaking fees, Lundbeck. M.H.M.: royalties, Allergan. L.L.I.: grants/grants pending, NIH 
NINDS. J.M.P.: grants/grants pending, NIH, VA, CURE.

\section{References}

1. Catterall WA, Dib-Hajj S, Meisler MH, Pietrobon D. Inherited neuronal ion channelopathies: new windows on complex neurological diseases. J Neurosci 2008;28:11768-11777.

2. Kullmann DM, Waxman SG. Neurological channelopathies: new insights into disease mechanisms and ion channel function. J Physiol 2010;588:1823-1827.

3. Dravet C, Bureau M, Oguni $H$, et al. Severe myoclonic epilepsy in infancy: Dravet syndrome. Adv Neurol 2005;95:71-102.

4. Guerrini R, Aicardi J. Epileptic encephalopathies with myoclonic seizures in infants and children (severe myoclonic epilepsy and myoclonic-astatic epilepsy). J Clin Neurophysiol 2003;20:449-461.

5. Claes L, Del-Favero J, Ceulemans B, et al. De novo mutations in the sodium-channel gene SCN1A cause severe myoclonic epilepsy of infancy. Am J Hum Genet 2001;68:1327-1332.

6. Meisler MH, Kearney JA. Sodium channel mutations in epilepsy and other neurological disorders. J Clin Invest 2005;115:20102017.

7. Kanai K, Hirose S, Oguni H, et al. Effect of localization of missense mutations in SCN1A on epilepsy phenotype severity. Neurology 2004;63:329-334

8. Ceulemans BP, Claes LR, Lagae LG. Clinical correlations of mutations in the SCN1A gene: from febrile seizures to severe myoclonic epilepsy in infancy. Pediatr Neurol 2004;30:236-243.

9. Catterall WA, Kalume F, Oakley JC. NaV1.1 channels and epilepsy. J Physiol 2010;588:1849-1859.

10. Spampanato J, Escayg A, Meisler MH, Goldin AL. Functional effects of two voltage-gated sodium channel mutations that cause generalized epilepsy with febrile seizures plus type 2. J Neurosci 2001;21:7481-7490.

11. Lossin C, Rhodes TH, Desai RR, et al. Epilepsy-associated dysfunction in the voltage-gated neuronal sodium channel SCN1A. J Neurosci 2003;23:11289-11295.

12. Yu FH, Mantegazza M, Westenbroek RE, et al. Reduced sodium current in GABAergic interneurons in a mouse model of severe myoclonic epilepsy in infancy. Nat Neurosci 2006;9:1142-1149.

13. Ogiwara I, Miyamoto $\mathrm{H}$, Morita $\mathrm{N}$, et al. $\mathrm{Na}(\mathrm{v}) 1.1$ localizes to axons of parvalbumin-positive inhibitory interneurons: a circuit basis for epileptic seizures in mice carrying an Scn1a gene mutation. J Neurosci 2007;27:5903-5914.

14. Takahashi K, Yamanaka S. Induction of pluripotent stem cells from mouse embryonic and adult fibroblast cultures by defined factors. Cell 2006;126:663-676.

15. Takahashi K, Tanabe K, Ohnuki M, et al. Induction of pluripotent stem cells from adult human fibroblasts by defined factors. Cell 2007;131:861-872.

16. Yu J, Vodyanik MA, Smuga-Otto K, et al. Induced pluripotent stem cell lines derived from human somatic cells. Science 2007;318:1917-1920.

17. Park IH, Arora N, Huo H, et al. Disease-specific induced pluripotent stem cells. Cell 2008;134:877-886.

18. Moretti A, Bellin M, Welling $A$, et al. Patient-specific induced pluripotent stem-cell models for long-QT syndrome. N Engl J Med 2010;363:1397-1409.

19. Itzhaki I, Maizels L, Huber I, et al. Modelling the long QT syndrome with induced pluripotent stem cells. Nature 2011;471:225229.
20. Yazawa $M$, Hsueh $B$, Jia $X$, et al. Using induced pluripotent stem cells to investigate cardiac phenotypes in Timothy syndrome. $\mathrm{Na}$ ture 2011;471:230-234.

21. Harkin LA, McMahon JM, lona $X$, et al. The spectrum of SCN1Arelated infantile epileptic encephalopathies. Brain 2007;130(pt 3):843-852.

22. Rusconi R, Scalmani P, Cassulini RR, et al. Modulatory proteins can rescue a trafficking defective epileptogenic Nav1.1 Na+ channel mutant. J Neurosci 2007;27:11037-11046.

23. Patino GA, Claes LR, Lopez-Santiago LF, et al. A functional null mutation of SCN1B in a patient with Dravet syndrome. J Neurosci 2009;29:10764-10778.

24. Fein AJ, Meadows LS, Chen C, et al. Cloning and expression of a zebrafish SCN1B ortholog and identification of a species-specific splice variant. BMC Genomics 2007;8:226.

25. Lopez-Santiago LF, Pertin M, Morisod X, et al. Sodium channel beta2 subunits regulate tetrodotoxin-sensitive sodium channels in small dorsal root ganglion neurons and modulate the response to pain. J Neurosci 2006;26:7984-7994.

26. Smith RD, Goldin AL. Functional analysis of the rat I sodium channel in xenopus oocytes. J Neurosci 1998;18:811-820.

27. Frankel WN. Genetics of complex neurological disease: challenges and opportunities for modeling epilepsy in mice and rats. Trends Genet 2009;25:361-367.

28. Erceg S, Laínez S, Ronaghi M, et al. Differentiation of human embryonic stem cells to regional specific neural precursors in chemically defined medium conditions. PLoS One 2008;3:e2122.

29. Kim JE, O'Sullivan ML, Sanchez CA, et al. Investigating synapse formation and function using human pluripotent stem cell-derived neurons. Proc Natl Acad Sci U S A 2011;108:3005-3010

30. Koch P, Opitz T, Steinbeck JA, et al. A rosette-type, self-renewing human ES cell-derived neural stem cell with potential for in vitro instruction and synaptic integration. Proc Natl Acad Sci U S A 2009;106:3225-3230.

31. Shi Y, Kirwan P, Livesey FJ. Directed differentiation of human pluripotent stem cells to cerebral cortex neurons and neural networks. Nat Protoc 2012;7:1836-1846.

32. Mantegazza M. Dravet syndrome: insights from in vitro experimental models. Epilepsia 2011;52(suppl 2):62-69.

33. Cheah CS, Yu FH, Westenbroek RE, et al. Specific deletion of NaV1.1 sodium channels in inhibitory interneurons causes seizures and premature death in a mouse model of Dravet syndrome. Proc Natl Acad Sci U S A 2012;109:14646-14651.

34. Mistry A, Miller A, Thompson C, et al. Strain and age-dependent differences in hippocampal neuron sodium current densities in a mouse model of Dravet Syndrome. Soc Neurosci Abstr 2012:G29:548.

35. Guerrini R, Striano P, Catarino C, Sisodiya SM. Neuroimaging and neuropathology of Dravet syndrome. Epilepsia 2011;52(suppl 2):30-34

36. Guerrini R, Dravet $C$, Genton $P$, et al. Lamotrigine and seizure aggravation in severe myoclonic epilepsy. Epilepsia 1998;39:508-512.

37. Dimos JT, Rodolfa KT, Niakan KK, et al. Induced pluripotent stem cells generated from patients with ALS can be differentiated into motor neurons. Science 2008;321:1218-1221.

38. Ebert AD, Yu J, Rose FF Jr, et al. Induced pluripotent stem cells from a spinal muscular atrophy patient. Nature 2009;457:277280.

39. Marchetto MC, Carromeu C, Acab A, et al. A model for neural development and treatment of Rett syndrome using human induced pluripotent stem cells. Cell 2010;143:527-539. 